\title{
ASSOCIATION BETWEEN THE WEIGHT LOSS AND ANXIETY, DEPRESSION AFTER PERCUTANEOUS TRANSLUMINAL CORONARY ANGIOPLASTY: A NEED FOR PRE-DISCHARGED COUNSELING
}

\author{
PRASAD N SHETTY ${ }^{1}$, DEVASIA TOM ${ }^{1}$, YESHWANTH RAO K ${ }^{2 *}$, AJIT SINGH $^{1}$, HASHEER KARIM ${ }^{1}$
}

${ }^{1}$ Department of Cardiology, Kasturba Hospital, Manipal University, Manipal - 576 104, Karnataka, India. ${ }^{2}$ Department of Pharmacology, Melaka Manipal Medical College (MMMC), Manipal University, Manipal - 576 104, Karnataka, India. Email: yashwanthrao2000@gmail.com

Received: 25 April 2017, Revised and Accepted: 16 May 2017

\section{ABSTRACT}

Objectives: To see the association between weight loss and anxiety, depression after percutaneous transluminal coronary angioplasty (PTCA) for coronary artery disease patients. (1) To see the severity of anxiety and depression 1 month after PTCA, (2) to evaluate the relationship between the weight changes and major adverse cardiac events (MACE) in 1 month follow-up.

Methods: A total 1001 patients who underwent PTCA for acute coronary syndrome and coronary heart disease were included. Data were collected at baseline and 1 month after PTCA. A validated Euro 5Q-5D-5L questionnaire was used to assess the level of anxiety and depression. Chi-square test was applied to find out the association between weight loss status and anxiety, depression.

Results: There was a significant decrease in mean weight 1 month after coronary angioplasty $(64.18 \pm 11.022$ vs. $61.96 \pm 11.17$, $p<0.001)$. No association was observed between weight loss status and anxiety, depression in PTCA patients $(\mathrm{p}=0.5)$ and there is no significant association between weight changes and MACE 1 month after PTCA $(\mathrm{p}=0.92)$.

Conclusion: Data show that anxiety and depression were observed in $64.9 \%$ of the study population after coronary angioplasty but anxiety and depression are not associated with weight loss after PTCA. Loss of weight may be due to the modified post angioplasty lifestyles such as adherent to Mediterranean diet, physical exercise, cessation of smoking, optimum control of blood pressure, blood sugar, and blood cholesterol along with prescribed medications. Anxiety and depression may turn into chronic stage if not treated wisely could lead to significant MACE. Proper pre-hospital discharge counseling is necessary for all post PTCA patients to sustain their quality of life.

Keywords: Percutaneous transluminal coronary angioplasty, Weight loss, Anxiety, Depression.

(C) 2017 The Authors. Published by Innovare Academic Sciences Pvt Ltd. This is an open access article under the CC BY license (http://creativecommons. org/licenses/by/4. 0/) DOI: http://dx.doi.org/10.22159/ajpcr.2017.v10i8.19393

\section{INTRODUCTION}

Maintaining healthy weight is one of the major concerns among the people. Increased body weight, being overweight, and obesity are considered as major risk factor for the acute myocardial infarction (AMI) [1]. American college of cardiology, American heart association and cardiologic societies recommend early detection wherein management of obesity is necessary for primary prevention because of the greater risk for adverse cardiac events and all-cause mortality in coronary artery disease (CAD) [2,3] There are many pathophysiological pathways by which obesity increases the risk for developing CAD and adverse cardiovascular events. Obesity reduces insulin sensitivity, enhances free fatty acid turnover, increases basal sympathetic tone, induces a hypercoagulable state, and promotes systemic inflammation, all of which contribute to the development and progression of CAD [4].

There are different modalities for losing weight, for example, starvation, liquid protein diets, very low-calorie diets, and even obesity surgeries [5]. Intentional and unintentional weight loss in post MI was observed, but there is no definite explainable mechanism for the loss of weight. Intentional weight loss, viz., switching into Mediterranean diet, use of omega-3 fatty acids, low cholesterol and low fatty acids diets, regular physical exercise, optimum control of blood sugar, and blood pressure may be protective in patients with CAD [6,7]

Unintentional weight loss also noted in post-operative surgeries including coronary artery bypass graft (CABG) surgeries and it is considered that restriction in the caloric intake in the post-operative period is vital cause for weight loss [8]. Apart from the surgeries there is an evidence of decreased weight in non-invasive procedure like percutaneous transluminal coronary angioplasty (PTCA) for CAD along with improved diet and exercise [9]. However, some effects were found on the different psychological variables, as well as on morbidity or return to work.

Depression and anxiety are common outcome after PTCA and have been linked with weight changes in the general population [10]. In animals, social isolation contributes to weight loss, and this may happen in humans $[11,12]$ asocial isolation is associated with worse outcomes after MI.

Unlike other psychosocial factors, depression and anxiety represent well-defined psychiatric disorders and they are important cause of coronary heart disease. Depression and coronary heart disease could share common backgrounds, for example, environmental stressors and social supports. Depressive episodes after MI have prognostic importance rather than severity of CAD [13]. Anxiety and depression may affect health-related behaviors such as smoking, tobacco abuse, alcohol consumption, diet, or physical activity, which in turn may influence the risk of coronary heart disease [14]. Many studies provide compelling evidence that psychosocial factors, particularly anxiety, depression and social support, are independent etiological and prognostic factors for occurrence of coronary heart disease. The relation between depression, anxiety and coronary heart disease differs from those of other psychosocial factors for several reasons. Depression and anxiety may cause direct acute or chronic pathophysiological changes. 
Anxiety, depression and associated disturbed quality of life (QoL) has been observed in a patient with PTCA for CAD. It is also noted that anxiety and depression are risk factors for the angina. Negative emotions play an important role in producing anxiety and depression [15]

Depression in cardiac patients is often both chronic and recurrent, and it is common among this patient, incidence is approximately $15 \%$ including those suffering acute coronary syndrome (ACS) and higher when compared to general population (4-5\%) or primary care patients (8-10\%) [16-18].

Depression and anxiety occur at high rates among patients suffering an ACS. Both depressive symptoms and anxiety appear to adversely affect in-hospital and long-term cardiac outcomes of post-ACS patients, independent of traditional risk factors. Despite their high prevalence and serious impact, mood and anxiety symptoms go unrecognized and untreated in most ACS patients and such symptoms (rather than being transient reactions to ACS) persist for months and beyond. The mechanisms by which depression and anxiety are linked to these negative medical outcomes are likely a combination of the effects of these conditions on inflammation, catecholamine's, heart rate variability, and endothelial function, along with effects on health promoting behavior [19-24].

Weight change after coronary angioplasty could affect the path of the disease, very few studies have assessed the effects of weight change on outcomes after coronary angioplasty. Furthermore, there are very few studies that have examined the role of psychosocial reaction such as depression and anxiety on this issue. Post-operative anxiety and depression are well established and are consider as psychological reactions which can see during as well as after the angioplasty [25]. However, there are no definite studies have been conducted to see the association between the post PTCA weight loss and post angioplasty behavioral changes such as anxiety and depression.

This analysis was undertaken to evaluate the magnitude, direction, and determinants of weight change following coronary angioplasty and its relationship to depression and anxiety.

\section{METHODS}

A total of 1001 patients between 18 and 65 years of age who underwent PTCA in Kasturba Medical College and Hospital, Manipal, Karnataka, India, for ACS as well as CAD were included in this prospective study. Patients with moderate or severe left ventricular dysfunction (ejection fraction $<40 \%$ ), cardiogenic shock on presentation/during hospitalization, chronic kidney disease, known psychiatric illness on medications diagnosed by physician and/or noted in the medical record, psychological disturbances and undergoing counseling or treatment for anxiety and depression, chronic liver disease, pregnant women, terminal malignancy patients with life expectation $<1$ year and patients with viral markers positive were excluded.

Details of the study were explained in patients own language and informed consent was taken from them. Demographic data that included age, gender, marital status, educational level, height, and weight were obtained. Body mass index (BMI) was calculated. To control for extraneous factors, we recorded smoking history, family history and pre-existing cardiovascular risk factors such as presence of diabetes, hypercholesterolemia, and hypertension. This medication and different bio-chemical reports were collected along with electrocardiogram, coronary angiogram, and coronary angioplasty (PTCA). The study was conducted prospectively, material and data were collected during hospitalization and on 1 month follow-up. Results were documented in a customized form or form designed by organization, containing complete details of patient's medications and investigations. On discharge, patient will be asked to come for follow-up on 1 month with investigation reports including fasting blood sugar, fasting lipid profile, and serum creatinine. On follow-up, documented the blood pressure and blood test reports of the patients and also assess the baseline characteristics including comorbidities, and anthropometry measurements, weight of the patients was measured. Assessment of psychological behavior such as anxiety and depression was done using the Euro groups standardized EQ-5D-5L-self-assessment QoL questionnaire downloaded from www. euroqol.org after getting prior permission. Euro-EQ-5D-5L contains five self-assessment questionnaire in anxiety/depression column reporting level 1 (no anxiety/depression), level 2 (mild anxiety/depression), level 3 (moderate anxiety/depression), level 4 (severe anxiety/depression), and level 5 (extreme anxiety/depression) [26].

\section{RESULTS}

There was significant decrease in mean body weight 1 month after PTCA (64.18 \pm 11.088 vs. $61.98 \pm 11.19, \mathrm{p}<0.001)$ (Table 1).

Psychological reactions like anxiety, depression after 1 month was $64.9 \%$, viz., mild anxiety, depression was $47.5 \%$, moderate anxiety, depression was $15.4 \%$ and severe/extreme anxiety, and depression was $2 \%$ of subjects. Among study population $71.81 \%$ subjects had lost their weight, $19.88 \%$ gained weight, and $8.31 \%$ had no changes in their weight 1 month after PTCA.

However, no significant association was found between weight changes and anxiety, depression in post coronary angioplasty patients $(\mathrm{p}=0.5)$ (Fig. 1).

In 1 month $7.8 \%$ of post PTCA patients had major adverse cardiac events (MACE) among this $2 \%$ had sudden cardiac death (SCD) and $5 \%$ had nonfatal MACE here also no association observed between weight change group and MACE ( $\mathrm{p}=0.92$ ) (Fig. 2), but there was a significant association between the anxiety, depression and MACE $(p<0.001)$ (Fig. 3).

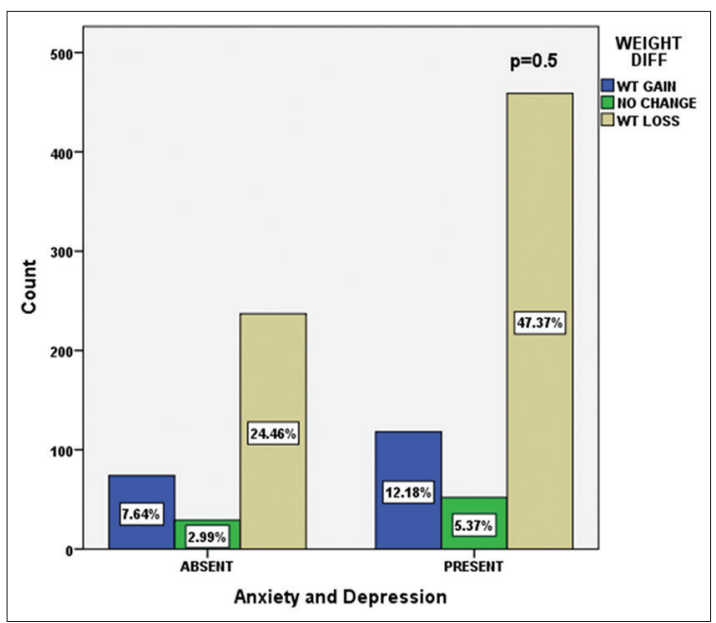

Fig. 1: Association between anxiety, depression and weight loss 1 month after coronary angioplasty

Table 1: Comparison of baseline and 1 month follow-up in overall study populations clinical and biochemical parameters

\begin{tabular}{llll}
\hline Parameters & \multicolumn{2}{l}{ Mean \pm SD } & \\
\cline { 2 - 4 } & Baseline & Follow-up & p value \\
\hline Weight $(\mathrm{kg})$ & $64.18 \pm 11$ & $61.9 \pm 11$ & $<0.001$ \\
BMI $\left(\mathrm{kg} / \mathrm{m}^{2}\right)$ & $23.8 \pm 4$ & $23.7 \pm 10$ & 0.671 \\
SBP $(\mathrm{mm}$ of $\mathrm{Hg})$ & $132 \pm 20$ & $131 \pm 20$ & 0.05 \\
DBP $(\mathrm{mm}$ of $\mathrm{Hg})$ & $82 \pm 12$ & $81 \pm 10$ & 0.371 \\
Glycosylated $\mathrm{Hb}$ & $7 \pm 2$ & $7 \pm 2$ & 0.017 \\
\hline
\end{tabular}

BMI: Body mass index, SBP: Systolic blood pressure, DBP: Diastolic blood pressure, SD: Standard deviation 


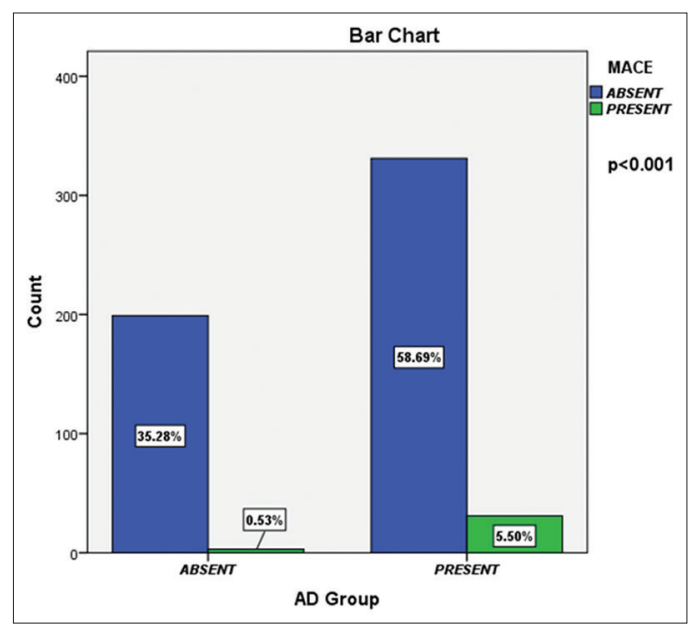

Fig. 2: Association between major adverse cardiac events and anxiety, depression 1 month post percutaneous transluminal coronary angioplasty

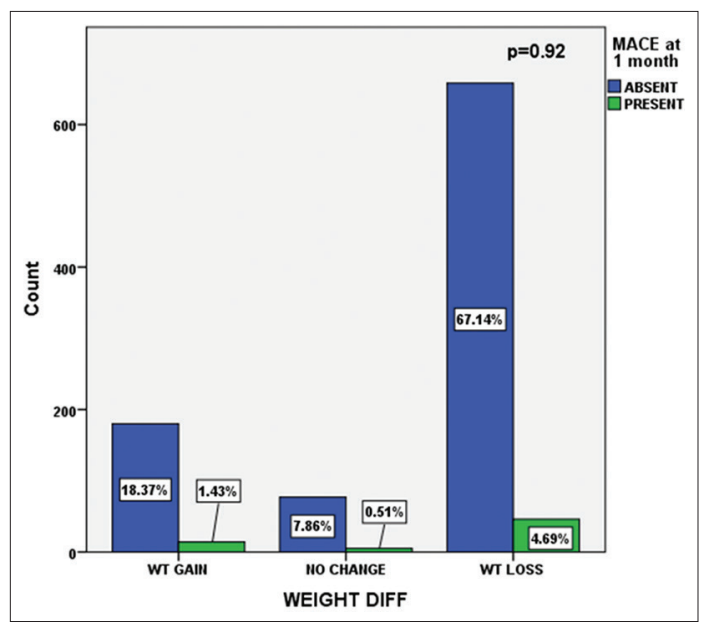

Fig. 3: Association between weight loss and major adverse cardiac events 1 month after coronary angioplasty

\section{DISCUSSION}

Many studies indicate that mean weight does not change after AMI $[27,28]$. However, in our study, there was a significant decrease in mean weight when compared to baseline weight; weight loss was associated with more comorbidities at baseline, suggesting weight loss might be a marker of coronary vascular disease (CVD), severity or poorer overall health. Weight loss may also be the result of lifestyle changes such as improved diet pattern, physical exercise, cessation of smoking/tobacco, optimum control blood cholesterol level, blood pressure, and blood sugar along with compliance with prescribed medication. Measurement of the rates of adherence to lifestyle changes and medications together with the identification of its determinants is of utmost importance for the design and provision of health education [29].

Although this study evidenced variation in weight and altered psychological reactions, there was no significant association found between these two outcomes. Weight loss also did not show significant association with MACE, whereas presence of anxiety, depression regardless of its severity had significant association with occurrence of MACE $(\mathrm{p}<0.01)$. This reveals that one's psychological status plays significant role in prognosis after PTCA, wherein predischarge counseling may have impact on psychological status.
Enriched cohort study explains that there is definite weight loss after MI but there is no association between psychological behavior such as depression and social support and weight loss. However, weight loss was not associated with SCD and nonfatal MI [30]. Our study also explains the same, there is no association found between SCD, nonfatal MI and weight loss. Remarkable weight loss was found in patients 1 year after bariatric surgery however, baseline depression and anxiety are not a predictors of post-surgical weight loss [31,32]. In addition, treating baseline anxiety/depression disorder might result in better QoL after surgery [32].

Post coronary angioplasty weight loss may be intentional or unintentional. If weight loss is intentional, it might be that awareness about post angioplasty health care and modification in lifestyle including healthy diet: Use of Mediterranean diet, omega-3 fatty acids, and physical exercise ( 30 minutes' walk at least 5 days in a week) are come into play [33].

Prospective cohort studies unhealthy populations strongly confirm that depression and anxiety are possible etiological and prognostic factor for coronary heart disease and adverse cardiac events like SCD [32].

Weight loss and weight gain are associated with depression and anxiety in non-cardiac area, most of antipsychotic medication induces obesity, is also leads to depression/anxiety, particularly if severe [34]. We hypothesized that depression, anxiety would be related to weight change in post coronary angioplasty patients because anxiety and depression are associated with adverse events after PTCA. However, depression, anxiety did not interact with weight loss at baseline or weight related rates of adverse events or survival.

Given the graded relation between depression scores and risk, the longlasting nature of the effect, and the stability of the depression measured across time, it has been proposed that depression is a continuously distributed chronic psychological characteristic and anxiety is part of it [35], hence it is important to treat the both dynamically to avoid post angioplasty risks.

\section{CONCLUSION}

In our study, post angioplasty weight loss was significant comparing to baseline and mild to moderate anxiety and depression noted in the study population, even though not associated with weight loss 1 month after coronary angioplasty, weight loss may be controlled post angioplasty diet, regular physical exercise, and cessation of smoking/tobacco. Even though no association found between weight loss and MACE in 1 month follow-up but long term basis weight loss may influence the MACE, there for, one should not lose their weight aggressively.

Depression and anxiety are believed to be indirect risk factors for MACE, we found significant association between MACE and anxiety, depression, this group patients are less likely to follow a low-fat diet, lack of interest in physical exercise, cessation of smoking/tobacco and to reduce their cholesterol, blood pressure, blood sugar or reduce stress in their lives and all of which increase their recurrent cardiac event. Depression and anxiety patients less likely to maintain their routine normal health and less likely to attend cardiac rehabilitation programs, both of which important to prevent secondary cardiac risk as well as adverse cardiac outcome. In sum, depression and anxiety have innumerable effects on cardiovascular physiology via different mechanism and may lead different cardiac pathology so proper counseling will be effective in this group of patient to maintain their long-term eventless post angioplasty health. It is hoped that factors which hinder a better understanding of patient counseling, shall be identified with the goal of improving the patient adherence/ compliance and satisfying today's need [36]. Several studies proved the importance of counseling to improve the patients' understanding and adherence to their prescribed medications [37]. 


\section{Limitations of the study}

Our findings underscore the need for clinical trials using these measures to test the effects of intentional or unintentional weight loss in patients with obesity and cardiovascular disease. We only used weight but waist-hip circumference is more identical to calculate the weight loss in cardiovascular disease. Our follow-up confined to only 1 month to see association between weight, anxiety and depression and to see the occurrence of the MACE.

\section{REFERENCES}

1. Lopez-Jimenez F, Jacobsen SJ, Reeder GS, Weston SA, Meverden RA, Roger VL. Prevalence and secular trends of excess body weight and impact on outcomes after myocardial infarction in the community. Chest 2004;125(4):1205-12.

2. Powell BD, Lennon RJ, Lerman A, Bell MR, Berger PB, Higano ST, et al. Association of body mass index with outcome after percutaneous coronary intervention. Am J Cardiol 2003;91(4):472-6.

3. Smith SC, Blair SN, Bonow RO. AHA/ACC guidelines for preventing heart attack and death in patients with atherosclerotic cardiovascular disease: 2001 update-a statement for healthcare professionals from the American heart association and the American college of cardiology. J Am Coll Cardiol 2001;38:1581-3.

4. Pi-Sunyer FX. The obesity epidemic: Pathophysiology and consequences of obesity. Obes Res 2002;10 Suppl 2:97S-104.

5. Poirier P, Giles TD, Bray GA, Hong Y, Stern JS, Pi-Sunyer FX, et al. Obesity and cardiovascular disease: Pathophysiology, evaluation, and effect of weight loss an update of the 1997 American Heart Association scientific statement on obesity and heart disease from the obesity committee of the council on nutrition, physical activity, and metabolism. Circulation 2006;113(6):898-918.

6. Pack QR, Rodriguez-Escudero JP, Thomas RJ, Ades PA, West CP, Somers VK, et al. The prognostic importance of weight loss in coronary artery disease: A systematic review and meta-analysis. Mayo Clin Proc 2014;89(10):1368-77.

7. de Lorgeril M, Salen P, Martin JL, Monjaud I, Delaye J, Mamelle N. Mediterranean diet, traditional risk factors, and the rate of cardiovascular complications after myocardial infarction: Final report of the Lyon Diet Heart Study. Circulation 1999;99(6):779-85.

8. Kocz1 R, Hassan MA, Perala PR, Negargar S, Javadzadegan H, Nader ND. The effect of weight loss on the outcome after coronary artery bypass grafting in obese patients. Ann Card Anaesth 2012;15(3):190-8.

9. Lisspers J, Sundin O, Hofman-Bang C, Nordlander R, Nygren A, Rydén L, et al. Behavioral effects of a comprehensive, multifactorial program for lifestyle change after percutaneous transluminal coronary angioplasty: A prospective, randomized, controlled study. J Psychosom Res 1999;46(2):143-54.

10. Wing RR, Phelan S. Long-term weight loss maintenance. Am J Clin Nutr 2005;82 1 Suppl:222S-5.

11. Yamada K, Ohki-Hamazaki H, Wada K. Differential effects of social isolation upon body weight, food consumption, and responsiveness to novel and social environment in bombesin receptor subtype-3 (BRS-3) deficient mice. Physiol Behav 2000;68(4):555-61.

12. van Leeuwen SD, Bonne OB, Avraham Y, Berry EM. Separation as a new animal model for self-induced weight loss. Physiol Behav 1997;62(1):77-81

13. Hemingway H, Marmot M. Evidence based cardiology: Psychosocial factors disease: Systematic review of prospective cohort. BMJ 1999;318(7196):1460-7

14. Booth JN $3^{\text {rd }}$, Levitan EB, Brown TM, Farkouh ME, Safford MM, Muntner P. Effect of sustaining lifestyle modifications (nonsmoking, weight reduction, physical activity, and mediterranean diet) after healing of myocardial infarction, percutaneous intervention, or coronary bypass (from the REasons for Geographic and Racial Differences in Stroke Study). Am J Cardiol 2014;113(12):1933-40.

15. Moattari M, Adib F, Kojuri J, Tabatabaee SH. Angina self-management plan and quality of life, anxiety and depression in post coronary angioplasty patients. Iran Red Crescent Med J 2014;16(11):e16981.

16. Rudisch B, Nemeroff CB. Epidemiology of comorbid coronary artery disease and depression. Biol Psychiatry 2003;54(3):227-40.

17. van Melle JP, de Jonge P, Spijkerman TA, Tijssen JG, Ormel J, van Veldhuisen DJ, et al. Prognostic association of depression following myocardial infarction with mortality and cardiovascular events: A meta-analysis. Psychosom Med 2004;66(6):814-22.

18. Glassman AH, Bigger JT, Gaffney M, Shapiro PA, Swenson JR. Onset of major depression associated with acute coronary syndromes: Relationship of onset, major depressive disorder history, and episode severity to sertraline benefit. Arch Gen Psychiatry 2006;63(3):283-8.

19. Grace SL, Abbey SE, Irvine J, Shnek ZM, Stewart DE. Prospective examination of anxiety persistence and its relationship to cardiac symptoms and recurrent cardiac events. Psychother Psychosom 2004;73(6):344-52.

20. Huffman JC, Smith FA, Blais MA, Beiser ME, Januzzi JL, Fricchione GL. Recognition and treatment of depression and anxiety in patients with acute myocardial infarction. Am J Cardiol 2006;98(3):319-24.

21. Kaptein KI, de Jonge P, van den Brink RH, Korf J. Course of depressive symptoms after myocardial infarction and cardiac prognosis: A latent class analysis. Psychosom Med 2006;68(5):662-8.

22. Barth J, Schumacher M, Herrmann-Lingen C. Depression as a risk factor for mortality in patients with coronary heart disease: A metaanalysis. Psychosom Med 2004;66(6):802-13

23. Frasure-Smith N, Lespérance F. Depression and anxiety as predictors of 2-year cardiac events in patients with stable coronary artery disease. Arch Gen Psychiatry 2008;65(1):62-71.

24. Berkman LF, Blumenthal J, Burg M, Carney RM, Catellier D, Cowan MJ, et al. Effects of treating depression and low perceived social support on clinical events after myocardial infarction: The Enhancing Recovery in Coronary Heart Disease Patients (ENRICHD) Randomized Trial. JAMA 2003;289(23):3106-16.

25. Huffman JC, Celano CM, Januzzi JL. The relationship between depression, anxiety, and cardiovascular outcomes in patients with acute coronary syndromes. Neuropsychiatr Dis Treat 2010;6:123-36.

26. van Hout B, Janssen MF, Feng YS, Kohlmann T, Busschbach J, Golicki D, et al. Interim scoring for the EQ-5D-5L: Mapping the EQ5D-5L to EQ-5D-3L value sets. Value Health 2012;15(5):708-15.

27. Lopez-Jimenez F, Wu CO, Tian X, O’Connor C, Rich MW, Burg MM, et al. Weight change after myocardial infarction - the Enhancing Recovery in Coronary Heart Disease patients (ENRICHD) experience. Am Heart J 2008;155(3):478-84

28. Dagenais GR, Yi Q, Mann JF, Bosch J, Pogue J, Yusuf S. Prognostic impact of body weight and abdominal obesity in women and men with cardiovascular disease. Am Heart J 2005;149(1):54-60.

29. Elbur AI. Level of adherence to lifestyle changes and medications among male hypertensive patients in two hospitals in Taif; Kingdom of Saudi Arabia. Int J Pharm Pharm Sci 2015;7(4):168-72.

30. Bucholz EM. Excess weight and life expectancy after acute myocardial infarction: The obesity paradox reexamined. Am Heart J 2016;172:173-81.

31. Shen R, Dugay G, Rajaram K, Cabrera I, Siegel N, Ren CJ. Impact of patient follow-up on weight loss after bariatric surgery. Obes Surg 2004;14(4):514-9.

32. Damien HS. Are Depression, Anxiety, Body Mass Index, and Types of Surgery Predictive of Weight Loss and Psychological Outcomes After Bariatric Surgery, Columbia University Academic Commons; 2012. Available from: http://www.hdl.handle.net/10022/ac:p:13067.

33. Sadeghzadeh V. Effects of a lifestyle-change program on cardiac risk factors after angioplasty. Int Res J Appl Basic Sci 2013;4(1):234-8.

34. Natali A, Vichi S, Landi P, Severi S, L'Abbate A, Ferrannini E. Coronary atherosclerosis in Type II diabetes: Angiographic findings and clinical outcome. Diabetologia 2000;43(5):632-41

35. Montgomery SA. Anxiety as part of depression. Acta Psychiatr Scand 1983;68:171-4.

36. Dola HS, Jadhava SS, Pisala MA, Shaikha SK, Shinde VR. Emerging trends in patient counselling: Current scenario. Int J Pharm Pharm Sci 2015;7(10):65-8

37. Khairunnisa NA, Tanjung HR. Impacts of counseling on adherence to prescribed medications and blood pressure of hypertensive patients in four Indonesian primary health centers. Int $\mathrm{J}$ Pharm Pharm Sci 2015;7(3):114-7. 